\title{
Finite valuated groups as modules over their endomorphism ring
}

\author{
Ulrich Albrecht
}

\begin{abstract}
This paper discusses the structure of a finite valuated $p$-group when viewed as a module over its endomorphism ring. A category equivalence between full subcategories of the category of valuated $p$-groups and the category of right modules over the endomorphism ring of $A$ is used to investigate the interaction between this module structure and homological properties of the underlying group. Examples are given throughout the paper.
\end{abstract}

Mathematics Subject Classification (2010): 20K30, 20K40, $20 \mathrm{~K} 10$.

Keywords: Valuated p-group, endomorphism ring, Ulmer's theorem, projective module.

\section{Introduction}

Consider a prime $p$ and a $p$-local Abelian group $G$. A valuation $v$ on $G$ assigns a value $v(g)$ to each $g \in G$ which is either an ordinal or $\infty$ subject to the rules

i) $v(p x)>v(x)$ for all $x \in G$ where $\infty>\infty$,

ii) $v(x+y) \geq \min \{v(x), v(y)\}$ for all $x, y \in G$, and

iii) $v(n x)=v(x)$ whenever $n$ and $p$ are relatively prime [11].

The third condition is redundant whenever $G$ is a $p$-group. The valuated $p$-local groups are the objects of the category $\mathcal{V}_{p}$ studied extensively by Hunter, Richman and Walker (e.g. see [7], [8] and [11]). A group homomorphism $\alpha:(G, v) \rightarrow(H, w)$ is a $\mathcal{V}_{p}$-morphism if $w(\alpha(x)) \geq v(x)$ for all $x \in G$, and we write $\alpha \in \operatorname{Mor}(G, H)$ in this case. The category $\mathcal{V}_{p}$ is pre-Abelian, i.e. all maps have kernels and cokernels. While the kernel and cokernel of a $\mathcal{V}_{p}$-map $G \rightarrow H$ are its kernel and cokernel in the category $\mathcal{A} b$ of Abelian groups, their valuations are induced by those on $G$ and $H$ respectively. Consequently, monomorphisms and epimorphisms need not be kernels and cokernels; and $\mathcal{V}_{p}$ is not Abelian. Finally, the forgetful functor $\mathcal{F}: \mathcal{V}_{p} \rightarrow \mathcal{A} b$ strips a valuated group $(G, v)$ of its valuation. 
In this paper, all valuated groups are assumed to be finite valuated $p$-groups. Although the group structure of a finite valuated $p$-group is well understood, the addition of a valuation directly impacts its homological properties. In addition, Arnold discovered a surprising connection between finite valuated $p$-groups and torsion-free Abelian groups of finite rank in [3] by demonstrating that representation theory can be used to investigate finite rank Butler groups as well as finite valuated $p$-groups. Moreover, both classes of groups are equally difficult to describe.

This paper follows Arnold's approach by investigating valuated $p$-groups using tools which have traditionally been used in the discussion of torsion-free groups of finite rank. For instance, homological properties of Abelian groups $A$ of finite torsionfree rank have been successfully studied by viewing $A$ as a left module over its endomorphism ring. This paper extends this approach to finite valuated $p$-groups by considering such a group $A$ as a module over its $\mathcal{V}_{p}$-endomorphism $\operatorname{ring} R=\operatorname{Mor}(A, A)$ and by studying how this module structure affects the homological properties of $A$. Section 2 focuses on the case that $A$ is projective as an $R$-module, while Section 3 considers the case that $R$ has specific ring-theoretic properties.

\section{Valuated $p$-Groups Projective as $R$-modules}

A finite valuated $p$-group $A$-free if it is isomorphic to $A^{n}$ for some $n<\omega$, and $A$-projective if it is a $\mathcal{V}_{p}$-direct summand of an $A$-free group. Since $A$ is a left $R$ module, $H_{A}=\operatorname{Mor}(A,-)$ can be viewed as a functor from $\mathcal{V}_{p}$ to the category $\mathcal{M}_{R}$ of right $R$-modules, with the property that $H_{A}(P)$ is free (projective) if $P$ is $A$-free (A-projective).

We begin our discussion with a few technical results. If $\alpha$ is a kernel in $\mathcal{V}_{p}$, then $\alpha=\operatorname{ker}(\operatorname{coker}(\alpha))[12]$; and a similar result holds for cokernels. However, composition of kernels (cokernels) in $\mathcal{V}_{p}$ need not be kernels (cokernels) [10]. Therefore, the usual homological constructions may not carry over from Abelian categories. Nevertheless, it is still possible to develop a homological algebra for pre-Abelian categories as Yakovlev showed in [14].

Lemma 2.1. Let $A, B$ and $C$ be valuated p-groups. If $\alpha \in \operatorname{Mor}(A, B)$ is an epimorphism and $\beta \in \operatorname{Mor}(B, C)$ such that $\beta \alpha$ is a cokernel of a $\mathcal{V}_{p}$-map $\delta$, then $\beta$ is a cokernel for $\alpha \delta$.

Proof. Suppose that $\phi$ satisfies $\phi \alpha \delta=0$. Since $\beta \alpha$ is a cokernel for $\delta$, there is a map $\psi$ such that $\psi \beta \alpha=\phi \alpha$. Because $\alpha$ is an epimorphism, $\phi=\psi \beta$. Since $\beta$ is an epimorphism, $\psi$ is unique with this property.

A sequence $A \stackrel{\alpha}{\rightarrow} B \stackrel{\beta}{\rightarrow} C$ of valuated $p$-groups is is left-exact if $\alpha$ is a kernel for $\beta$, and right-exact if $\beta$ is a cokernel for $\alpha$. It is exact in $\mathcal{V}_{p}$ if $\alpha$ is a kernel for $\beta$ and $\beta$ is a cokernel for $\alpha$ [11]. The functor $H_{A}: \mathcal{V}_{p} \rightarrow \mathcal{M}_{R}$ is left-exact since

$$
0 \rightarrow H_{A}(U) \stackrel{H_{A}(\alpha)}{\longrightarrow} H_{A}(B) \stackrel{H_{A}(\beta)}{\longrightarrow} H_{A}(C) \quad(*)
$$


is an exact sequence of right $R$-modules whenever

$$
0 \rightarrow U \stackrel{\alpha}{\longrightarrow} B \stackrel{\beta}{\longrightarrow} C
$$

is a left-exact sequence of valuated $p$-groups.

Consider the functor $t_{A}: \mathcal{M}_{R} \rightarrow \mathcal{A} b$ defined by $t_{A}=-\otimes_{R} A$ for all $M \in \mathcal{M}_{R}$. If $F$ is a free right $R$-module with basis $\left\{x_{i} \mid i \in I\right\}$, then

$$
v\left(\Sigma_{i \in I} x_{i} \otimes a_{i}\right)=\min \left\{v\left(a_{i}\right) \mid i \in I\right\}
$$

defines a valuation on $t_{A}(F)$, and the resulting valuated group is denoted by $T_{A}(F)$ [1]. To define a valuation on $t_{A}(M)$ for an arbitrary right $R$-module $M$, we choose a free resolution

$$
F_{1} \stackrel{\alpha}{\longrightarrow} F_{0} \stackrel{\beta}{\longrightarrow} M \rightarrow 0
$$

of $M$. Applying $t_{A}$ induces an exact sequence

$$
T_{A}\left(F_{1}\right) \stackrel{t_{A}(\alpha)}{\longrightarrow} T_{A}\left(F_{0}\right) \stackrel{t_{A}(\beta)}{\longrightarrow} t_{A}(M) \rightarrow 0
$$

where $t_{A}(\alpha)$ is a $\mathcal{V}_{p}$-map, which we denote as $T_{A}(\alpha)$, by [1]. Since $\mathcal{V}_{p}$ is pre-Abelian, there is a unique valuation $v$ on $t_{A}(M)$ such that $t_{A}(\beta)$ becomes the $\mathcal{V}_{p}$-cokernel of $T_{A}(\alpha)$ [11]. We define $T_{A}(M)=\left(t_{A}(M), v\right)$, and observe $t_{A}=\mathcal{F} T_{A}$. The next result summarizes the basic properties of $T_{A}$ which were established in [2, Section 2]:

Theorem 2.2. [2] Let $A$ be a finite valuated p-group.

a) $T_{A}: \mathcal{M}_{R} \rightarrow \mathcal{V}_{p}$ is a right exact functor.

b) The evaluation map $\theta_{G}: T_{A} H_{A}(G) \rightarrow G$ defined by $\theta_{G}(\alpha \otimes a)=\alpha(a)$ is a natural $\mathcal{V}_{p}$-map for all valuated p-groups $G$ such that $\theta_{P}$ is an isomorphism for all A-projective groups $P$.

c) The natural map $\Phi_{M}: M \rightarrow \operatorname{Hom}\left(A, T_{A}(M)\right)$ defined by $\left[\Phi_{M}(x)\right](a)=x \otimes a$ is a natural transformation such that $\theta_{T_{A}(M)} T_{A}\left(\Phi_{M}\right)=1_{T_{A}(M)}$ for all right $R$ modules $M$. Moreover, $\Phi_{P}$ is an isomorphism for all finitely generated projective right $R$-modules $P$.

An epimorphism $G \rightarrow H$ of valuated $p$-groups is $A$-balanced if the induced map $H_{A}(\alpha): H_{A}(G) \rightarrow H_{A}(H)$ is onto. A valuated $p$-group $G$ is weakly $A$-generated if we can find an $A$-balanced epimorphism

$$
\oplus_{I} A \stackrel{\beta}{\longrightarrow} G \rightarrow 0
$$

for some index-set $I$. It is $A$-generated if $\beta$ can be chosen to be a cokernel in $\mathcal{V}_{p}$. Although there is no need to distinguish between $A$-generated and weakly $A$-generated objects in an Abelian category, it is necessary to do this in the pre-Abelian case as was shown in [2].

A valuated $p$-group $G$ is $A$-presented if there is an exact sequence

$$
0 \rightarrow U \rightarrow F \rightarrow G \rightarrow 0
$$

of valuated $p$-groups such that $F$ is $A$-free and $U$ is weakly $A$-generated. If this sequence can be chosen to be $A$-balanced, then $G$ is called $A$-solvable. A valuated p-group $G$ is $A$-presented if and only if $G \cong T_{A}(M)$ for some right $R$-module $M$. 
Moreover, it is $A$-solvable if and only if $\theta_{G}$ is an isomorphism [2]. In particular, every $A$-projective group is $A$-solvable.

In a pre-Abelian category like $\mathcal{V}_{p}$, neither the 5-Lemma nor the Snake-Lemma need to hold [11]. The next result is frequently used in this paper as a substitute for the 5-Lemma throughout this paper:

Lemma 2.3. Let $A$ be a finite valuated p-groups. If $0 \rightarrow U \stackrel{\alpha}{\longrightarrow} H \stackrel{\beta}{\longrightarrow} G \rightarrow 0$ is a $\mathcal{V}_{p}$-exact sequence such that $\theta_{H}$ is an isomorphism, then there exists a commutative $\mathcal{V}_{p}$-diagram

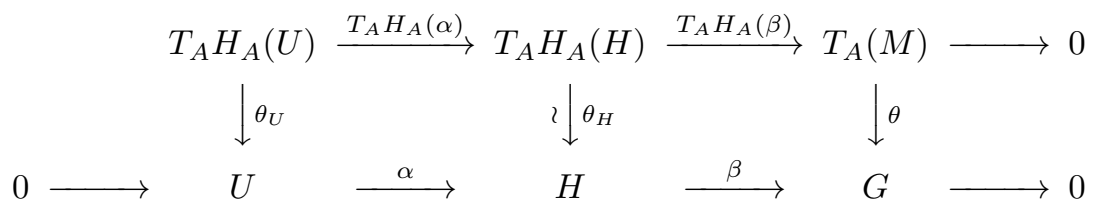

with $\mathcal{V}_{p}$-exact rows in which $M=i m H_{A}(\beta) \subseteq H_{A}(G)$ and $\theta: T_{A}(M) \rightarrow G$ is the evaluation map. Moreover, $\theta$ is a cokernel, and $\theta=\theta_{G} T_{A}(\iota)$ where $\iota: M \rightarrow H_{A}(G)$ is the inclusion map.

Proof. Since $H_{A}$ is left-exact, every exact sequence

$$
0 \rightarrow U \stackrel{\alpha}{\longrightarrow} H \stackrel{\beta}{\longrightarrow} G \rightarrow 0
$$

of valuated groups induces an exact sequence

$$
0 \rightarrow H_{A}(U) \stackrel{H_{A}(\alpha)}{\longrightarrow} H_{A}(H) \stackrel{H_{A}(\beta)}{\longrightarrow} M \rightarrow 0
$$

of right $R$-modules where $M=i m\left(H_{A}(\beta)\right)$ is a submodule of $H_{A}(G)$. By Part a) of Theorem 2.2, the induced sequence

$$
T_{A} H_{A}(U) \stackrel{T_{A} H_{A}(\alpha)}{\longrightarrow} T_{A} H_{A}(H) \stackrel{T_{A} H_{A}(\beta)}{\longrightarrow} T_{A}(M) \rightarrow 0
$$

is right exact. Part b) of same result yields that $\theta_{U}$ and $\theta_{G}$ are $\mathcal{V}_{p}$-maps, and the commutativity of the diagram follows directly. Since $T_{A}(\iota)$ is a $\mathcal{V}_{p}$-map by another application of Theorem 2.2, the same holds for $\theta=T_{A}(\iota) \theta_{G}$. Using the fact that $\theta_{H}$ is a $\mathcal{V}_{p}$-isomorphism, we obtain $\theta\left[T_{A}(\beta) \theta_{H}^{-1}\right]=\beta$. Because $T_{A}(\beta)$ is a cokernel, $\theta$ is a cokernel by Lemma 2.1 .

Ulmer described the objects of an Abelian Groethendick category which are flat over their endomorphism ring [13]. When discussing the validity of Ulmer's result in $\mathcal{V}_{p}$, one immediately realizes that his original arguments need to be modified extensively because this category is only pre-Abelian. In particular, we want to remind the reader that a finite valuated $p$-group is flat as an $R$-module if and only if it is projective.

Theorem 2.4. The following conditions are equivalent for a finite valuated $p$-group A:

a) $A$ is projective as a left $R$-module.

b) Whenever $\phi \in \operatorname{Mor}\left(A^{n}, A\right)$ for some $n<\omega$, then ker $\phi$ is weakly A-generated.

c) Whenever $\phi \in \operatorname{Mor}(G, H)$ for $A$-solvable valuated $p$-groups $G$ and $H$, then $\operatorname{ker} \phi$ is weakly A-generated. 
Proof. $a) \Rightarrow c)$ : For $K=\operatorname{ker} \phi$, consider the exact sequence

$$
0 \rightarrow H_{A}(K) \longrightarrow H_{A}(G) \stackrel{\phi}{\longrightarrow} M \rightarrow 0
$$

of right $R$-modules in which $M=i m\left(H_{A}(\phi)\right)$ is a submodule of $H_{A}(H)$. Let $\iota$ denote embedding $M \subseteq H_{A}(H)$. By Proposition 2.3, we obtain a commutative diagram

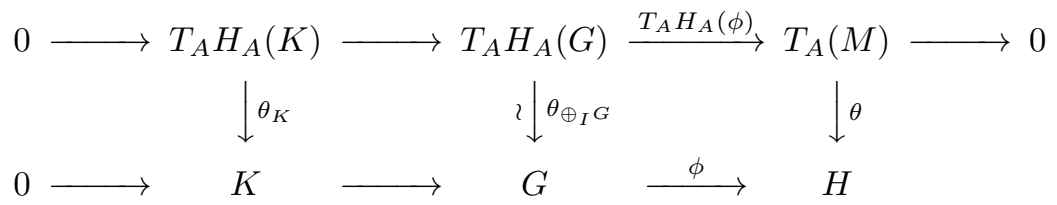

of $\mathcal{V}_{p}$-maps whose top-row is right exact in $\mathcal{V}_{p}$. Moreover, it is exact in $\mathcal{A} b$ since $A$ is projective as a left $R$-module. Using the projectivity of $A$ once more yields that $T_{A}(\iota)$ is a monomorphism, and the same holds for $\theta=\theta_{H} T_{A}(\iota)$ since $H$ is $A$-solvable. Thus, $\theta$ is an isomorphism of Abelian groups. Because the 3-Lemma is valid in $\mathcal{A} b$, we obtain that $\theta_{K}$ is an epimorphism in $\mathcal{A} b$, and hence in $\mathcal{V}_{p}$.

Since $c) \Rightarrow b$ ) is obvious, it remains to show $b) \Rightarrow a$ ):

It suffices to establish that the inclusion map $\iota: I \rightarrow R$ induces a monomorphism $t_{A}(\iota): t_{A}(I) \rightarrow t_{A}(R)$ of Abelian groups for all right ideals $I$ of $R$. Since $R$ is finite, $I=\left\{r_{1}, \ldots, r_{n}\right\}$. We define a map $\phi_{1}: F=R^{n} \rightarrow I$ by $\phi_{1}\left(e_{i}\right)=r_{i}$ where $\left\{e_{1}, \ldots, e_{n}\right\}$ is an $R$-basis of $F$. Set $\phi=\iota \phi_{1}: F \rightarrow R$. By b), the kernel $K$ of the $\mathcal{V}_{p}$-map $T_{A}(\phi): T_{A}(F) \rightarrow T_{A}(R)$ is weakly $A$-generated. Since $A$ is finite, we can select a finite $A$-projective group $P$ and an $A$-balanced epimorphism $\lambda: P \rightarrow K$. Because

$$
0 \rightarrow K \rightarrow T_{A}(F) \stackrel{T_{A}(\phi)}{\longrightarrow} T_{A}(R)
$$

is $\mathcal{V}_{p}$-exact, the induced sequence

$$
0 \rightarrow H_{A}(K) \rightarrow H_{A} T_{A}(F) \stackrel{H_{A} T_{A}(\phi)}{\longrightarrow} H_{A} T_{A}(R)
$$

is exact. Combining this sequence with $H_{A}(\lambda)$ yields that the top-row of the commutative diagram

$$
\begin{aligned}
& H_{A}(P) \stackrel{H_{A}(\lambda)}{\longrightarrow} H_{A} T_{A}(F) \stackrel{H_{A} T_{A}(\phi)}{\longrightarrow} H_{A} T_{A}(R)
\end{aligned}
$$

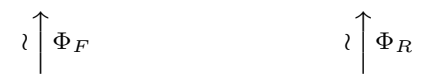

$$
\begin{aligned}
& F \stackrel{\phi}{\longrightarrow} \quad R
\end{aligned}
$$

of right $R$-modules is exact. In view of $\phi(F)=I$, the diagram gives us the exact sequence

$$
\text { (E) } H_{A}(P) \stackrel{H_{A}(\lambda)}{\longrightarrow} H_{A} T_{A}(F) \stackrel{\phi_{1} \Phi_{F}^{-1}}{\longrightarrow} I \rightarrow 0
$$

of right $R$-modules. Since $\theta_{T_{A}(M)} T_{A}\left(\Phi_{M}\right)=1_{T_{A}(M)}$ for all right $R$-modules $M$, we obtain $\theta_{T_{A}(X)}=T_{A}\left(\Phi_{X}^{-1}\right)$ for all finitely generated projective right $R$-modules $X$. Hence,

$$
T_{A}(\phi) \theta_{T_{A}(F)}=T_{A}\left(\phi \Phi_{F}^{-1}\right)=T_{A}\left(\Phi_{R}^{-1} H_{A} T_{A}(\phi)\right)=\theta_{T_{A}(R)} T_{A} H_{A} T_{A}(\phi) .
$$

Because of this and Theorem 2.2, an application of $T_{A}$ yields the commutative diagram 


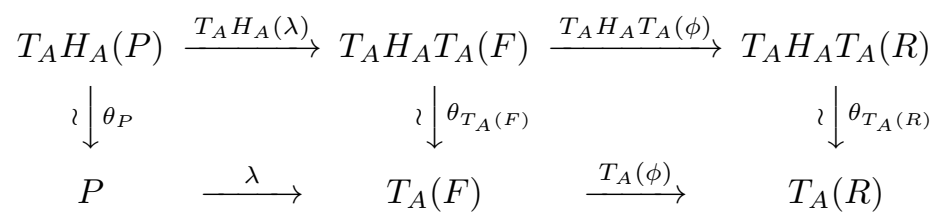

of Abelian groups. Since it suffices to show that $t_{A}(\iota)$ is a monomorphism of Abelian groups, our computations are done from this point only in $\mathcal{A} b$ instead of in $\mathcal{V}_{p}$. In particular, we use the fact that the $\mathcal{V}_{p}$-kernel of a map is its kernel in $\mathcal{A} b$ with a valuation added. The symbols $t_{A}$ and $T_{A}$ can be used interchangeably when computing in $\mathcal{A} b$.

Observe that the bottom row of the last diagram is exact at $T_{A}(F)$ as a sequence of Abelian groups by the choice of $P$ and $\lambda$. Since the vertical maps are isomorphisms, the top-row is exact at $T_{A} H_{A} T_{A}(F)$. Moreover, $(E)$ induces the exact sequence

$$
T_{A} H_{A}(P) \stackrel{T_{A} H_{A}(\lambda)}{\longrightarrow} T_{A} H_{A} T_{A}(F) \stackrel{T_{A}\left(\phi_{1} \Phi_{F}^{-1}\right)}{\longrightarrow} T_{A}(I) \rightarrow 0
$$

of Abelian groups. Therefore, the map $T_{A}\left(\phi_{1} \Phi_{F}^{-1}\right)$ is a cokernel in $\mathcal{A} b$ for the left top-map $T_{A} H_{A}(\lambda)$. On the other hand, the projection

$$
\pi: T_{A}(F) \rightarrow G=T_{A}(F) / K
$$

is a cokernel of $\lambda$ in $\mathcal{A} b$. Hence, there is an isomorphism $\sigma: T_{A}(I)=t_{A}(I) \rightarrow G$ of Abelian groups such that $\pi \theta_{T_{A}(F)}=\sigma T_{A}\left(\phi_{1} \Phi_{F}^{-1}\right)$. Since the bottom row of the last diagram is exact at $T_{A}(F)$, there is a map $\tau: G \rightarrow T_{A}(R)$ with $\tau \pi=T_{A}(\phi)$ using the exactness of the bottom row of the last diagram once more. For $g \in \operatorname{ker} \tau$, select $x \in T_{A}(F)$ with $\pi(x)=g$. Then $0=\tau \pi(x)=T_{A}(\phi)(x)$ yields $x=\lambda(y)$ for some $y \in P$. Hence, $g=\pi \lambda(y)=0$, and $\tau$ is a monomorphism.

Because $H_{A} T A\left(\phi_{1}\right) \Phi_{F}=\Phi_{I} \phi_{1}$, we have

$$
\begin{aligned}
\theta_{T_{A}(R)} T_{A} H_{A} T_{A}(\iota) T_{A}\left(\Phi_{I}\right) T_{A}\left(\phi_{1}\right) & =\theta_{T_{A}(R)} T_{A} H_{A} T_{A}(\iota) T_{A} H_{A} T_{A}\left(\phi_{1}\right) T_{A}\left(\Phi_{F}\right) \\
& =\theta_{T_{A}(R)} T_{A} H_{A} T_{A}(\phi) T_{A}\left(\Phi_{F}\right) \\
& =T_{A}(\phi) \theta_{T_{A}(F)} T_{A}\left(\Phi_{F}\right) \\
& =\tau \pi \theta_{T_{A}(F)} T_{A}\left(\Phi_{F}\right) \\
& =\tau \sigma T_{A}\left(\phi_{1} \Phi_{F}^{-1}\right) T_{A}\left(\Phi_{F}\right) \\
& =\tau \sigma T_{A}\left(\phi_{1}\right) .
\end{aligned}
$$

Since $T_{A}\left(\phi_{1}\right)$ is an epimorphism, we obtain that

$$
\theta_{T_{A}(R)} T_{A} H_{A} T_{A}(\iota) T_{A}\left(\Phi_{I}\right)=\tau \sigma
$$

is a monomorphism since the maps on the right are monomorphisms, and the same holds for

$$
T_{A}\left(\Phi_{R}\right) t_{A}(\iota)=T_{A} H_{A} T_{A}(\iota) T_{A}\left(\Phi_{I}\right)
$$

using the fact that $T_{A}(R) \cong A$. Because $T_{A}\left(\Phi_{R}\right)$ is an isomorphism, $t_{A}(\iota)$ is one-to-one as desired.

For a finite $p$-group $G$, let $e(A)$ denote the smallest $n<\omega$ such that $p^{n} G=0$. 
Corollary 2.5. Every finite valuated p-group $A$ is a direct summand of a finite valuated p-group $B$ such that $e(A)=e(B)$ and $B$ is flat as a module over its endomorphism ring.

Proof. Choose $n<\omega$ minimal with the property that $p^{n} A=0$, and consider the group $B=\mathbb{Z} / p^{n} \mathbb{Z} \oplus A$ where $\mathbb{Z} / p^{n}$ carries the height valuation $h$. Since $h$ is the smallest valuation on $\mathbb{Z} / p^{n} \mathbb{Z}$, and every $B$-generated group is bounded by $p^{n}$, the kernel of every map between any two $B$-generated groups is a $\mathcal{V}_{p}$-epimorphic image of $\left(\mathbb{Z} / p^{n} \mathbb{Z}, h\right)$. By Theorem $2.4, B$ is projective over its endomorphism ring.

We continue our discussion by looking at simply presented groups. A ( $p$-)valuated tree is a set $X$, on which a partial multiplication by $p$ is defined, together with a function $v$ assigning a value $v(x)$ to each $x \in X$ which is either an ordinal or $\infty$ subject to the rules

i) If $p^{n} x=x$ for some $0<n<\omega$, then $p x=x$, and there is exactly one element in $X$ with this property, called the root of $X$.

ii) $v(p x)>v(x)$ whenever $p x$ is defined.

Moreover, if $X_{1}, \ldots, X_{n}$ are rooted valuated trees, then the co-product $\cup_{i=1}^{n} X_{i}$ in the category of valuated $p$-tree is the tree that is obtained by joining $X_{1}, \ldots, X_{n}$ at their roots.

Associated with any rooted tree $X$ is a simply presented valuated $p$-group $S(X)$ defined as $F_{X} / R_{X}$ where $F_{X}$ is a free $\mathbb{Z}_{p}$-module with basis $\{\langle x\rangle \mid x \in X\}$ and $R_{X}$ is generated by the elements $p\langle x\rangle-\langle p x\rangle$. If we set $\bar{x}=\langle x\rangle+R_{X}$, then every $g \in S(X)$ has a unique presentation $g=\Sigma_{x \in X} n_{x} \bar{x}$ with $0 \leq n_{x}<p$, and the valuation on $S(X)$ is defined by

$$
v(g)=\min \left\{v(x) \mid n_{x} \neq 0\right\} .
$$

Finally, a valuated cyclic $p$-group $G$ of order $p^{n}$ is of the form $G=S(X)$ for a valuated $p$-tree $X=\left\{x_{0}, \ldots, x_{n-1}\right\}$ such that $G=\left\langle x_{0}\right\rangle$ and $x_{i}=p x_{i-1}$ for $i=1, \ldots, n$.

A map $\psi: X \rightarrow Y$ between valuated trees is a tree map if $\psi(p x)=p \psi(x)$ if $p x$ exists and $v(\psi(x)) \geq v(x)$. A tree map $r: X \rightarrow X$ is a retraction if $r^{2}=r$. Hunter, Richman and Walker showed that there is an order preserving retraction from $S(X)$ onto $X$ for all valuated trees [7]. Moreover, every tree map $\psi: X \rightarrow Y$ induces a $\mathcal{V}_{p}$-map $\bar{\psi}: S(X) \rightarrow S(Y)$.

Corollary 2.6. The following conditions are equivalent for a finite valuated $p$-group A:

a) $A$ is a cyclic group.

b) $A$ is an indecomposable simply presented group which is projective as an $R$ module.

Proof. It remains to show that an indecomposable simply presented group $A$ is cyclic if it is projective as an $R$-module. Since $A$ is indecomposable, $R$ is a local ring. Therefore, all projective $R$-modules are free. Consequently, we can find $a \in A$ such that $A=R a$, and $r a \neq 0$ for all non-zero $r \in R$.

Write $A=S(X)$ for some valuated tree $X$. Since $A$ is indecomposable, $X$ is irretractable and has a unique element $y$ of order $p$. Let $x_{1}, \ldots, x_{n}$ be the elements of maximal order of $X$, and select $r_{1}, \ldots, r_{n} \in R$ such that $x_{i}=r_{i} a$ for $i=1, \ldots, n$. 
If $r_{1}, \ldots, r_{n} \in J(R)$, then $A=J(R) A$ because $x_{1}, \ldots, x_{n}$ generate $A$ as an Abelian group, which is impossible by Nakayama's Lemma. Therefore, we may, without loss of generality, assume $r_{1} \notin J(R)$. Thus, $r_{1}$ is a unit in $R$, and

$$
A=R a=R r_{1} a=R x_{1} .
$$

Moreover, if $s x_{1}=0$, then

$$
0=s x_{1}=s r_{1}\left(r_{1}^{-1} x_{1}\right)=s r_{1} a
$$

from which we obtain $s r_{1}=0$. Then $s=0$ since $r_{1}$ is a unit of $R$. Therefore, $\phi\left(x_{1}\right) \neq 0$ for all non-zero $\phi \in R$.

Suppose that $n>1$, and define a map $r: X \rightarrow X$ by $r(x)=0$ if $x \neq x_{2}$ and $r\left(x_{2}\right)=y$. Observe that $v\left(x_{2}\right) \leq v(y)$ by the choice of $x_{2}$ and $y$. For $x \neq x_{2}, p x \neq x_{2}$ because $x_{2}$ is an element of maximal order. Thus, $r(p x)=0$. On the other hand $\operatorname{pr}\left(x_{2}\right)=p y=0$ while $r\left(p x_{2}\right)=0$ since $p x_{2} \neq x_{2}$. Therefore, $r$ is a map of valuated trees, and induces an endomorphism $\alpha$ of the valuated group $A$ with $\alpha\left(x_{1}\right)=0$ and $\alpha\left(x_{2}\right)=y \neq 0$, a contradiction. Consequently, $X$ has only one element $x_{1}$ of maximal order, and $A=\left\langle x_{1}\right\rangle$.

However, Corollary 2.5 shows that a simply presented group which is flat as a module over its endomorphism ring need not be a direct sum of cyclic groups. Moreover, there are infinitely many isomorphism classes of indecomposable finite valuated p-groups $G$ such that $p^{4} G=0$ and $v(g) \leq 9$ for all $0 \neq g \in G$ [3, Example 8.2.5]. Furthermore, the category of indecomposable finite valuated $p$-groups $G$ such that $p^{5} G=0$ and $v(g) \leq 11$ for all $0 \neq g \in G$ has wild representation type [3, Example 8.2.6].

Example 2.7. Let $A_{1}=\left\langle a_{1}\right\rangle, A_{2}=\left\langle a_{2}\right\rangle$ and $A_{3}=\left\langle a_{3}\right\rangle$ be cyclic groups of order $p^{3}$, and define a valuation on $A_{1}$ by $v\left(a_{1}\right)=1, v\left(p a_{1}\right)=4$ and $v\left(p^{2} a_{1}\right)=5$ and on $A_{2}$ by $v\left(a_{2}\right)=2, v\left(p a_{2}\right)=3$ and $v\left(p^{2} a_{2}\right)=5$. Finally, set $v\left(a_{3}\right)=\infty$.

To see that $A=A_{1} \oplus A_{2} \oplus A_{3}$ is not flat as an $R$-module, consider the map $\delta: A_{1} \oplus A_{2} \rightarrow A_{3}$ defined by $\delta\left(\left(n a_{1}, m a_{2}\right)\right)=(n-m) a_{3}$. It is easy to see that $K=\operatorname{ker} \delta=<\left(a_{1}, a_{2}\right)>$ and $v\left(a_{1}, a_{2}\right)=1, v\left(p a_{1}, p a_{2}\right)=3$, and $v\left(p^{2} a_{1}, p^{2} a_{2}\right)=5$.

If $\phi \in \operatorname{Mor}\left(A_{1}, K\right)$, then $\phi\left(a_{1}\right) \in p K$ for otherwise

$$
4=v\left(p a_{1}\right) \leq v\left(\phi\left(p a_{1}\right)\right)=v\left(p a_{1}, p a_{2}\right)=3 .
$$

Similarly, if $\psi \in \operatorname{Mor}\left(A_{2}, K\right)$, then $\psi\left(a_{2}\right) \in p K$ since otherwise

$$
2=v\left(a_{2}\right) \leq v\left(\psi\left(a_{2}\right)\right)=v\left(a_{1}, a_{2}\right)=1 .
$$

Since $\operatorname{Mor}\left(A_{3}, A_{1} \oplus A_{2}\right)=0$, we have $\operatorname{im} \theta_{K} \subseteq p K$, and $K$ is not weakly $A$-generated. By Theorem 2.4, $A$ is not projective as an $R$-module.

Example 2.8. If $A=\langle x\rangle$ is a cyclic group of order $p^{2}$ with the height valuation, then $A$ is free as a module over its endomorphism ring $E=\mathbb{Z} / p^{2} \mathbb{Z}$. Moreover, $v(p x)=1$. On the other hand, $M=\mathbb{Z} / p \mathbb{Z}$ is a left $E$-module which fits into the exact sequence

$$
E \stackrel{\alpha}{\longrightarrow} E \stackrel{\beta}{\longrightarrow} M \rightarrow 0
$$

where $\alpha\left(1+p^{2} \mathbb{Z}\right)=p+p^{2} \mathbb{Z}$ and $\beta\left(1+p^{2} \mathbb{Z}\right)=1+p \mathbb{Z}$. Then $T_{A}(M) \cong \mathbb{Z} / p \mathbb{Z}$ and setting $v(1+p \mathbb{Z})=0$ yields the cokernel valuation on $T_{A}(M)$. On the other hand, 
the map $\gamma: M \rightarrow E$ defined by $\gamma(1+p \mathbb{Z})=p+p^{2} \mathbb{Z}$ induces a monomorphism $T_{A}(\gamma): T_{A}(M) \rightarrow A$ such that $i m\left(T_{A}(\gamma)\right)=\langle p x\rangle$. Since

$$
0=v(1+p \mathbb{Z})<v(p x)=1
$$

the map $T_{A}(\gamma)$ does not preserve valuations. If we consider the sequence

$$
0 \rightarrow M \stackrel{\gamma}{\rightarrow} E \stackrel{\beta}{\longrightarrow} M \rightarrow 0,
$$

then $T_{A}(\gamma): T_{A}(M) \rightarrow T_{A}(E)$ is not a kernel for $T_{A}(\beta)$.

Therefore, the class of $A$-solvable groups may behave quite different from the case that $A$ is either a torsion-free or mixed Abelian group even if $A$ is a finite valuated $p$-group which is projective over its endomorphism ring. For instance, the kernel of a map between two $A$-solvable groups need not be $A$-solvable, nor is a weakly $A$ generated subgroup $U$ of an $A$-solvable group necessarily $A$-solvable.

Corollary 2.9. Let $A$ be a finite valuated p-group which is projective as an $R$-module. An A-generated subgroup $U$ of an A-solvable group $G$ is $A$-solvable.

Proof. By Proposition 2.3, it remains to show that $\theta_{U}$ is an isomorphism in $\mathcal{V}_{p}$. Since $A$ is projective as an $R$-module, one can argue as in the case of torsion-free groups that $\theta_{U}$ is an isomorphism of Abelian groups. Select an $A$-free group $F$ and an $A$-balanced exact sequence $0 \rightarrow V \stackrel{\alpha}{\longrightarrow} F \stackrel{\beta}{\longrightarrow} U \rightarrow 0$. It induces the commutative diagram

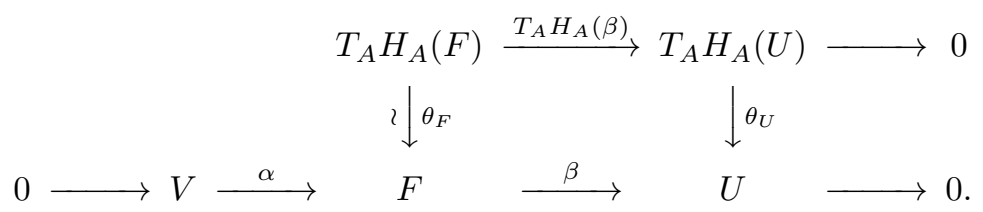

Since $\theta_{U}$ is an isomorphism of Abelian groups, $T_{A} H_{A}(\beta) \theta_{F}^{-1} \alpha=0$. There is a $\mathcal{V}_{p}$-map $\lambda: U \rightarrow T_{A} H_{A}(U)$ such that $T_{A} H_{A}(\beta) \theta_{F}^{-1}=\lambda \beta$ because $\beta$ is a cokernel of $\alpha$ in $\mathcal{V}_{p}$. Then

$$
\theta_{U} \lambda \beta=\theta_{U} T_{A} H_{A}(\beta) \theta_{F}^{-1}=\beta
$$

yields $\theta_{U} \lambda=1_{U}$. Thus, $\lambda \theta_{U}=1_{T_{A} H_{A}(U)}$ since $\theta_{U}$ is an isomorphism of Abelian groups. Hence

$$
v(x)=v\left(\lambda \theta_{U}(x)\right) \geq v\left(\theta_{U}(x)\right) \geq v(x)
$$

for all $x \in T_{A} H_{A}(U)$. Thus, $\theta_{U}$ is a $\mathcal{V}_{p}$-isomorphism.

Corollary 2.10. The following conditions are equivalent for a finite valuated p-group A:

a) $A$ is a progenerator for ${ }_{R} \mathcal{M}$.

b) i) Whenever $\phi \in \operatorname{Mor}(G, H)$ for $A$-solvable valuated $p$-groups $G$ and $H$, then ker $\phi$ is weakly A-generated.

ii) Whenever $\phi \in \operatorname{Mor}(G, H)$ is an epimorphism of A-solvable valuated $p$ groups $G$ and $H$, then $H_{A}(\phi)$ is an epimorphism. 
Proof. $a) \Rightarrow b$ ): It remains to show that ii) holds. For this, consider the submodule $M=i m H_{A}(\phi)$ of $H_{A}(H)$, and denote the inclusion map $M \rightarrow H_{A}(H)$ by $\iota$. The evaluation map $\theta: T_{A}(M) \rightarrow H$ is a $\mathcal{V}_{p}$-map since it satisfies $\theta=\theta_{H} T_{A}(\iota)$. Moreover, it is one-to-one since $A$ is a projective as a right $R$-module guarantees that $T_{A}(\iota)$ is a monomorphism of Abelian groups and $\theta_{H}$ is an isomorphism. On the other hand, it also fits into the commutative diagram

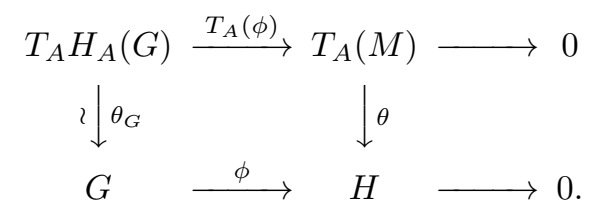

Hence, $\theta$ is an isomorphism of Abelian groups, and the same holds for $T_{A}(\iota)$. However, the latter fits into the exact sequence

$$
T_{A}(M) \stackrel{T_{A}(\iota)}{\longrightarrow} T_{A} H_{A}(H) \rightarrow H_{A}(H) / M \rightarrow 0 .
$$

Therefore, $T_{A}\left(H_{A}(H) / M\right)=0$. Since $A$ is a projective generator, $M=H_{A}(H)$.

b) $\Rightarrow a$ ): By [9, Proposition 2.4], every faithful projective module is a generator. Since $A$ is a projective left $R$-module by Theorem 2.4, it remains to show that it is faithful. Let $M$ be a right $R$-module with $t_{A}(M)=0$, and consider an exact sequence $P \rightarrow F \rightarrow M \rightarrow 0$ in which $P$ and $F$ are projective module. By Theorem 2.2, we obtain a right exact sequence $T_{A}(P) \rightarrow T_{A}(F) \rightarrow 0$ of valuated $p$-groups. By ii), the top sequence in the diagram

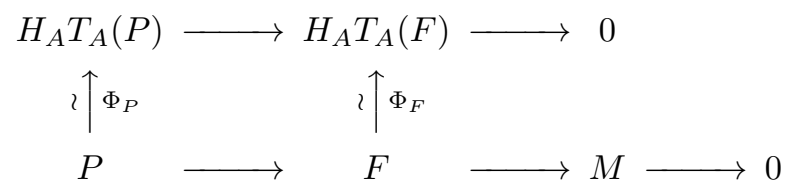

is exact. Thus, $M=0$.

\section{Hereditary and Quasi-Frobenius Endomorphism Rings}

We conclude our discussion by considering finite valuated $p$-groups $A$ whose endomorphism ring has specific ring-theoretic properties. We focus particularly on the cases that $R$ is either hereditary or self-injective. We want to remind the reader that there is no need to deal with right/left conditions since $R$ is finite [4].

A finite valuated $p$-group $G$ is $A$-torsion-less if there is a monomorphism $G \rightarrow A^{\ell}$ for some $\ell<\omega$. We say that an exact sequence of valuated groups is $A$-cobalanced if $A$ is injective with respect to it.

Theorem 3.1. Let $R$ be a finite valuated p-group A:

a) $R$ is hereditary if and only if $A$ is a direct sum of cyclic groups of order $p$.

b) $R$ is (semi-)simple Artinian if and only if $A \cong B^{m}$ where $B$ is a cyclic group of order $p$. 
c) If $R$ is a quasi-Frobenius ring, then every exact sequence $0 \rightarrow U \rightarrow G$ in which $U$ is weakly A-generated and $G$ is $A$-solvable is $A$-cobalanced. If $A$ is a projective $R$-module, then the converse holds, and every A-presented group is A-torsionless.

Proof. a) If $R$ is hereditary, then so is $e R e$ for any idempotent $e$ of $R$ [4]. If $B$ is an indecomposable summand of $A$, then there is a primitive idempotent $e$ of $R$ such that $e R e$ is the $\mathcal{V}_{p}$-endomorphism ring of $B$. Since $e R e$ is a hereditary local ring, all right ideals of $e R e$ are free $e R e$-modules. However, this means that $e R e$ is a field since it is finite. Because, $p E(B)$ is a proper ideal of $E(B)$, we have $p B=0$. By [8], $B$ is a cyclic group. Hence, $A$ is a direct sum of cyclic groups of order $p$.

Conversely, if $A$ has the described form, then $A=A_{1} \oplus \ldots \oplus A_{n}$ where $A_{i} \cong B_{i}^{\ell_{i}}$ and each $B_{i}$ is a cyclic group of order $p$. If $B_{i}=\left\langle b_{i}\right\rangle$, then no generality is lost if we assume $v\left(b_{i}\right)<v\left(b_{j}\right)$ for $i<j$ and $v\left(b_{i}\right) \neq \infty$ for $i<n$. Then $\operatorname{Mor}\left(B_{i}, B_{j}\right) \cong \mathbb{Z} / p \mathbb{Z}$ if $i \leq j$, and $\operatorname{Mor}\left(B_{i}, B_{j}\right)=0$ otherwise. Therefore, $R$ is Morita-equivalent to a lower triangular matrix ring over $\mathbb{Z} / p \mathbb{Z}$. By [5], $R$ is hereditary.

b) We continue using the notation from a). If $A=A_{1} \oplus \ldots \oplus A_{n}$ and $n>1$, then $\operatorname{Mor}\left(A_{i}, A_{j}\right)=0$ for $i>j$, but $\operatorname{Mor}\left(A_{i}, A_{j}\right) \neq 0$ for $i<j$. In particular, $N(R) \neq 0$. b) now follows immediately.

c) If $R$ is quasi-Frobenius, then we consider an exact sequence $0 \rightarrow U \stackrel{\alpha}{\longrightarrow} G$ in which $U$ is an epimorphic image of an $A$-projective group and $G$ is $A$-solvable. For $\phi \in \operatorname{Mor}(U, A)$, we can find a map $\psi: H_{A}(G) \rightarrow R$ such that $\psi H_{A}(\alpha)=\phi$. Since both, $\alpha$ and $\phi$, fit into the commutative diagram

we obtain

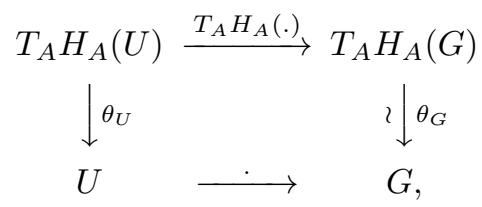

$$
T_{A}(\psi) \theta_{G}^{-1} \alpha \theta_{U}=\theta_{A} T_{A}(\psi) T_{A} H_{A}(\alpha)=\theta_{A} T_{A} H_{A}(\phi)=\phi \theta_{U} .
$$

Because $\theta_{U}$ is a $\mathcal{V}_{p}$-epimorphism, $T_{A}(\psi) \theta_{G}^{-1} \alpha=\phi$.

Conversely, let

$$
0 \rightarrow I \stackrel{\alpha}{\longrightarrow} R
$$

be an exact sequence and $\phi \in \operatorname{Hom}_{R}(I, R)$. Because $A$ is a flat $R$-module,

$$
0 \rightarrow T_{A}(I) \stackrel{T_{A}(\alpha)}{\longrightarrow} T_{A}(R)
$$

is a $\mathcal{V}_{p}$-exact sequence. Since $T_{A}(I)$ is an image of an $A$-projective group, there is a map $\psi \in \operatorname{Mor}\left(T_{A}(R), T_{A}(R)\right)$ such that $\psi T_{A}(\alpha)=T_{A}(\phi)$. We consider commutative diagrams of the form

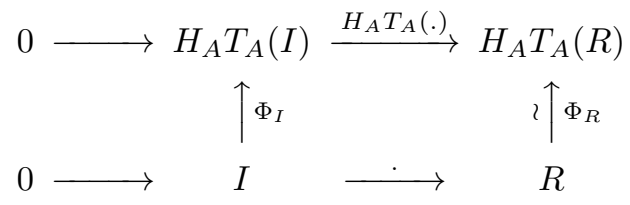


to obtain

$$
\begin{aligned}
\Phi_{R}^{-1} H_{A}(\psi) \Phi_{R} \alpha & =\Phi_{R}^{-1} H_{A}(\psi) H_{A} T_{A}(\alpha) \Phi_{I} \\
& =\Phi_{R}^{-1} H_{A} T_{A}(\phi) \Phi_{I} \\
& =\Phi_{R}^{-1} \Phi_{R} \phi=\phi .
\end{aligned}
$$

Finally, if $G$ is an $A$-presented group, then $G \cong T_{A}(M)$ for some finitely generated right $R$-module $M$ by [2] as mentioned before. Let $E$ be an injective hull of $M$. Since $R$ is quasi-Frobenius, $E$ is projective. Thus, $M$ can be embedded into a free $R$-module $F$, which can be chosen to be finite since $M$ is finite. Then $T_{A}(M)$ is isomorphic to a submodule of $T_{A}(F)$ since $A$ is projective.

Corollary 3.2. Let $A$ be a finite valuated p-group whose endomorphism ring is selfinjective. Every exact sequence

$$
0 \rightarrow P \stackrel{\alpha}{\longrightarrow} G
$$

such that $P$ is A-projective and $G$ is A-solvable splits.

We conclude with two examples that show that the endomorphism ring of a direct sum of cyclic valuated $p$-groups may or may not be quasi-Frobenius:

Example 3.3. a) Let $A_{1}$ be a cyclic group of order $p^{n}$, and $A_{2}$ a cyclic valuated group of order $p^{n}$ whose generator $x$ satisfies $v\left(p^{n-1} x\right)>n$. Then, the endomorphism ring of $A=A_{1} \oplus A_{2}$ is the lower triangular matrix ring over $\mathbb{Z} / p^{n} \mathbb{Z}$, which is not self-injective.

b) By $[6$, Example 1], the ring

$$
R=\left[\begin{array}{cc}
\mathbb{Z} / p^{3} \mathbb{Z} & p \mathbb{Z} / p^{3} \mathbb{Z} \\
p \mathbb{Z} / p^{3} \mathbb{Z} & \mathbb{Z} / p^{3} \mathbb{Z}
\end{array}\right]
$$

is quasi-Frobenius. Consider two cyclic valuated groups $A_{1}=\left(\left\langle x_{1}\right\rangle, v_{1}\right)$ and $A_{2}=$ $\left(\left\langle x_{2}\right\rangle, v_{2}\right)$ of order $p^{3}$ such that $v_{1}\left(x_{1}\right)=1, v_{1}\left(p x_{1}\right)=4, v_{2}\left(x_{2}\right)=2, v_{2}\left(p x_{2}\right)=3$ and $v_{1}\left(p^{2} x_{1}\right)=v_{2}\left(p^{2} x_{2}\right) \geq 5$. In view of the fact that $\operatorname{Mor}\left(A_{i}, A_{j}\right) \cong \mathbb{Z} / p^{2} \mathbb{Z}$ for $i \neq j$, we obtain that $A=A_{1} \oplus A_{2}$ has $R$ as its $\mathcal{V}_{p}$-endomorphism ring.

\section{References}

[1] Albrecht, U., Bewertete p-Gruppen und ein Satz von Szele, J. Algebra, 97(1985), 201-220.

[2] Albrecht, U., A-presented valuated groups, preprint.

[3] Arnold, D.M., Abelian Groups and Representations of Finite Partially Ordered Sets, CMS Books in Mathematics, Springer, 2000.

[4] Chatters, A.W., Hajarnavis, C.R., Rings with Chain Conditions, Pitman, 1980.

[5] Goodearl, K.R., Ring Theory, Pure and Applied Mathematics, No. 33, Marcel Dekker, New York - Basel, 1976.

[6] Hanulla, T.A., On the construction of quasi-Frobenius rings, J. Algebra, 25(1973), 403414.

[7] Hunter, R., Richman, F., Walker, E.A., Simply presented valuated p-groups, J. Algebra, 49(1977), 125-133. 
[8] Hunter, R., Richman, F., Walker, E.A., Finite direct sums of cyclic valuated p-groups, Pacific J. Math., 69(1977), 97-133.

[9] Ishikawa, T., Faithfully exact functors and their applications to projective modules and injective modules, Nagoya Math. J., 44(1964), 29-42.

[10] Richman, F., Walker, E.A., Ext in pre-Abelian categories, Pacific J. Math., 71(1977), 521-535.

[11] Richman, F., Walker, E.A., Valuated groups, J. Algebra, 56(1979), 145-167.

[12] Stenstrom, Bo, Rings of Quotients, Die Grundlehren der Mathematischen Wissenschaften, Band 217, Springer-Verlag, New York - Heidelberg, 1975.

[13] Ulmer, F., A flatness criterrion in Groethendick categories, Invent. Math., 19(1973), 331-336.

[14] Yakolev, A.V., Homological algebra in pre-abelian categories, Journal of Soviet Mathematics, 19(1982), 1060-1067.

Ulrich Albrecht

Department of Mathematics

Auburn University

Auburn, AL 36849, U.S.A.

e-mail: albreuf@mail . auburn.edu 